\section{Diode-Free Architecture Promises Dramatic Increase in Memory Density in Magnetic Random Access Memory Devices}

Magnetic random access memories (MRAMs) utilize the orientation of magnetization of a thin ferromagnetic film to store digital information in a nonvolatile manner. Currently, this requires the coupling of a diode (the selective component) in series with a magnetic tunnel junction (MTJ, the storage node). Unfortunately, the surface area of the typical diode used

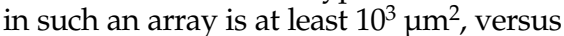
approximately $0.17 \mu^{2}$ for the smallest MTJ. The diode is thus the limiting factor in determining the storage density of an MRAM device.

In the September 25 issue of Applied Physics Letters, Frank Z. Wang of the School of Informatics and Multimedia Technology at the University of North London reported the development of a diode-free MRAM architecture. Since the absence of diodes means that all junctions of the MTJ array share a mutual electrical connection, it would seem to be impossi- ble to read the status of any given MTJ without including the contributions from the rest of them in the array. Wang solved this problem by introducing a "virtual ground,"a concept used in operational amplifiers, into a $2 \times 2$ bit memory "chiplet." Because the input impedance of an operational amplifier is very high or infinite, no current can flow into or out of the inverting input terminals, creating a virtual ground at $0 \mathrm{~V}$. MTJs were fabricated as a sandwich by deposition of a $100 \mathrm{~nm}$ layer of $\mathrm{Co}$, followed by a 3-8 nm layer of $\mathrm{Al}_{2} \mathrm{O}_{3}$, topped with a $100 \mathrm{~nm}$ layer of $\mathrm{NiFe}$, by sputtering with argon. The four MTJs in the 2 times 2 grid were designated $\mathrm{Bit}_{11}, \mathrm{Bit}_{12}, \mathrm{Bit}_{21}$, and $\mathrm{Bit}_{22}$, with each subscript referring to the corresponding word line and bit line, respectively, of the conducting paths at the nearest intersection. Setting word line 2 to an input excitation voltage while clamping bit line 2 to virtual ground through a read-mode switch caused the status of Bit ${ }_{22}$, and only $\mathrm{Bit}_{22}$, to appear at the output of the operational amplifier, thus proving the diodefree MRAM concept. According to Wang, the greatly improved memory density this development promises could boost the use of MRAM devices over semiconductor memories or magnetic disks in the future.

TIM PALUCKA

\section{Stable, Guest-Free Clathrate Form of Crystalline Silicon Synthesized}

A team of researchers from Jacksonville State University in Alabama and Arizona State University in Tempe, Arizona has reported the synthesis of a guest-free clathrate form of crystalline silicon. Optical and electrical measurements indicate that the properties of these structures are in reasonable agreement with existing theories predicting such new forms of crystalline silicon, with a wide $(1.9 \mathrm{eV})$ and indirect bandgap.

Silicon clathrates were first obtained as metastable intermediate phases during thermal decomposition of alkali metal silicides. Previous forms of these silicon clathrates required the presence of clumps of alkali or alkaline earth metals as "guest" materials to prevent collapse of the struc-

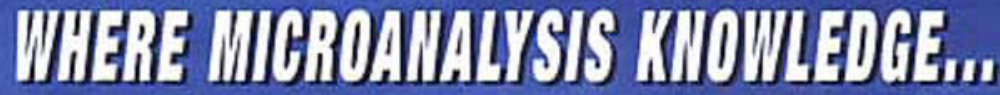

SUPERIOR PRODUCTS,

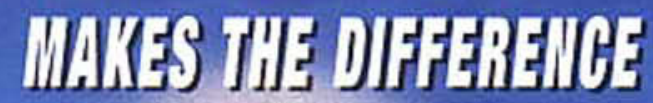
TRAINING AND SUPPORTIJ

$\Delta$ Wide range of high performance detecting units

$\Delta$ Diverse number of integrated system offerings

$\Delta$ Comprehensive range of upgrade paths

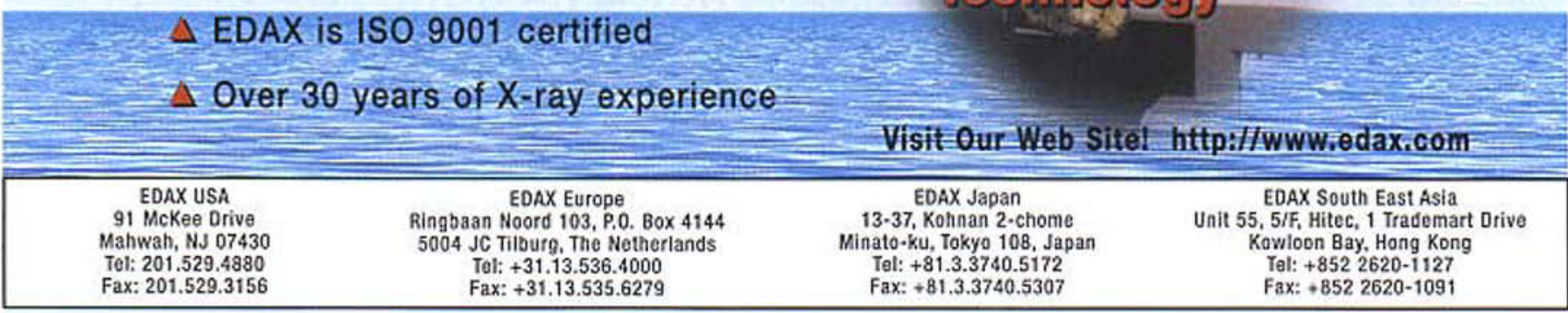

